\title{
Performance optimization of paste thickening
}

\author{
Mika Kosonen Outotec, Finland \\ Sakari Kauvosaari Outotec, Finland \\ Shan Gao Outotec, Finland \\ Brandt Henriksson Outotec, Australia
}

\section{Abstract}

Traditionally, thickener controls are implemented as single loop controllers in plant control systems. Based on our experiences from numerous thickener installations, this approach has several drawbacks, leading easily to poor process control and sub-optimal process performance. Especially challenging applications are paste thickeners, where the requirement is to achieve consistently high underflow density and high system availability without suffering process upsets and mechanical failures.

The main reason for the poor performance of the traditional, single loop Proportional Integral (PI) controllers is their inability to handle the slow response dynamics and cross action between controlled variables. This makes tuning of the PI-loops challenging, and compromises have to be made between system robustness and the desired speed of response. In many cases, these controllers are run either totally or partially in manual mode, and operator intervention is needed to maintain process stability and the desired operation point in varying running situations.

This paper discusses the optimizing control solution for paste thickeners. Initially, the instrumentation needed is briefly discussed. After this, an optimizing control solution based on multivariable model predictive control $(M P C)$ is introduced, and the results from applying this control strategy to a production scale concentrate thickener are presented. Finally, a simulated example shows how the same control strategy improves the performance of paste thickener when compared to traditional control strategies. It is shown that by using an advanced, model based control strategy, the thickener can consistently run with higher and more stable underflow density, so that the required level of overflow clarity is also obtained.

\section{Introduction}

Traditionally, thickener controls are implemented as single loop controllers in DCS/PLC systems. Based on our experiences from numerous thickener installations around the world, we have identified the following main challenges in this approach:

- Thickeners in general suffer from poor process control, resulting in sub-optimal performance.

- In many cases, controllers are run either totally or partially in manual mode.

- Operator intervention is needed to maintain the desired operation point in varying running situations.

- Excessive amounts of polymers are used as a precaution to compensate for changes in material settling properties.

The main reason for this is that traditional single-loop PI controllers are not optimal for handling the behavior of the thickening process. Slow and complicated response dynamics and cross-actions between the controlled variables make PI loops very challenging to tune, and compromises must be made between system 
robustness and the desired response speed. This paper discuss the optimizing control solution for paste thickeners. Initially, the instrumentation needed is briefly discussed. After this, an optimizing control solution based on multivariable model predictive control (MPC) is introduced, and the results from applying this control strategy to a production scale concentrate thickener are presented. Finally, a simulated example shows how the same control strategy improves the performance of the paste thickener when compared to traditional control strategies.

\section{Process instrumentation}

Robust and reliable process instrumentation is the cornerstone of a control system. The instrumentation installed should be suitable for the process, and it should be operated and maintained in an appropriate manner. This chapter discusses, on a general level, the key process instrument utilized for paste thickener process control. These measurements include density, the inventory level, overflow turbidity and rake torque measurements (Parker et al., 2016).

\subsection{Density measurement}

Achieving the target underflow density is a major key performance indicator for a thickener, and hence it should be measured online. Even if automatic control of density is not used, the relationship between bed mass and underflow density tells an experienced operator the efficiency of dewatering process. Density measurements are quite rarely used in the incoming lines due to relatively high investment. However, when they are applied especially together with incoming flow measurement, they provide valuable information for the control system designer about the changes in the feed dry mass flow. While a range of instruments is available for density measurement, gamma radiation-based instruments represent the industry standard and are widely available.

\subsection{Thickener inventory level}

Thickeners accumulate solids to create the bed pressure necessary to achieve dewatering. During steady state operation, the long-term solids withdrawal rate should match the feed rate. To achieve this steady state of operation, it is desirable to either measure both mass flows with a high level of accuracy, or measure the solids inventory in the thickener tank. However, accurate mass flow reconciliation between inflow and outflow is difficult due to instrument error, leading to uncertainty. Therefore, direct measurement of inventory level is normally the preferred solution.

Basically, two different approaches exist for inventory measurement: the first is based on the measurement of the hydrostatic pressure in the bottom cone of the thickener - i.e., bed pressure. Second approach aims for the direct detection of the height of the formed bed of solids.

\subsubsection{Bed pressure}

In bed pressure measurement, inventory indication is achieved by means of a pressure transducer mounted at the bottom of the tank. This bed pressure is measured in $\mathrm{kPa}$, but can be transformed to "\% bed mass" units for easier interpretation by operators. Best practice has the sensor flush mounted to the inside of the tank, because any buildup of solids forming a barrier between the bed and the sensor will reduce the accuracy of the reading. When installed properly, bed mass is a very robust and reliable measurement, and hence a good choice for process control.

\subsubsection{Bed level}

Typical techniques used for bed level measurements are either based on ultrasonic sonar or diver probes entering into the thickener. Both systems have their own benefits and challenges in implementation. Implementation of the sonar-based system is rather straightforward, because the sensor is installed on top 
of the thickener. However, they are sensitive to situations where the sonar response changes according to process condition and re-calibration of the measurement is needed.

Alternatively, diver probes are not sensitive to free solids material, so they are suitable for paste applications having cloudy overflow and a bed level line within the cylindrical section of the thickener. However, due to their mechanical nature, rather complicated and costly measurement setup is needed for fully automated probing systems.

\subsection{Overflow turbidity}

Turbidity is measured by detecting the attenuation of light when it passes through a water sample. It is a relative measurement for the amount of solids and colloidal particles in suspension and hence it can be used to detect the clarity of thickener overflow. As such, it does not directly tell the solids content, but it can be used as a "watch-dog" to avoid the escape of solids to the overflow.

\subsection{Rake torque}

High levels of rake torque are required in modern thickeners due to design and operational improvements that allow increased underflow density targets. These torque levels can have catastrophic consequences, unless control and protection systems are in place. From process control point of view, it is important that monitoring of the rake torque is included in the control strategy, so that system optimization is able to prevent situations leading to excessive torque levels.

\section{$3 \quad$ Optimizing control for thickeners}

In this chapter, a novel approach to utilize a multivariable, model predictive controller (MPC) in thickeners is presented. MPC is an advanced process control method which has been used in chemical plants and oil refineries since 1980s. In recent years, it has spread among other process industries also, and nowadays it is considered to be a standard method for control processes with a multivariable character and complex response dynamics (Maciejowski, 2002; Kosonen, 2004).

MPC control is based on the process response models, which are typically obtained by performing process trials or simulating the process responses. These process models are used to predict process behavior in the future. Based on this prediction, a set of future control actions is calculated to drive the process to the desired setpoints.

\subsection{System structure}

The optimizing control solution thickener optimizer is built on the Outotec Advanced Control Tool (ACT) platform, and the controller structure is shown in Figure 1. The controlled variables in the system are:

- Underflow density.

- Overflow turbidity.

- Thickener inventory level (bed pressure).

The control manipulates the underflow pump speed and flocculant dosing rate. Information from the thickener feed line, input flow rate and slurry density can also be used as feed-forward information when available. Rake torque is also taken into account as a constraint in the optimization, so that control actions leading to critical rake loads are avoided.

In multivariable MPC, there is no direct manipulated variable versus controlled variable relationships, meaning that one specific actuator, like underflow pump speed, is adjusted purely to control one specific controlled variable, e.g. underflow density. Instead, controller outputs are calculated as an optimized result of the cost function, which take into account the predicted control errors, predicted actuator movements and constraints given to the controller. 


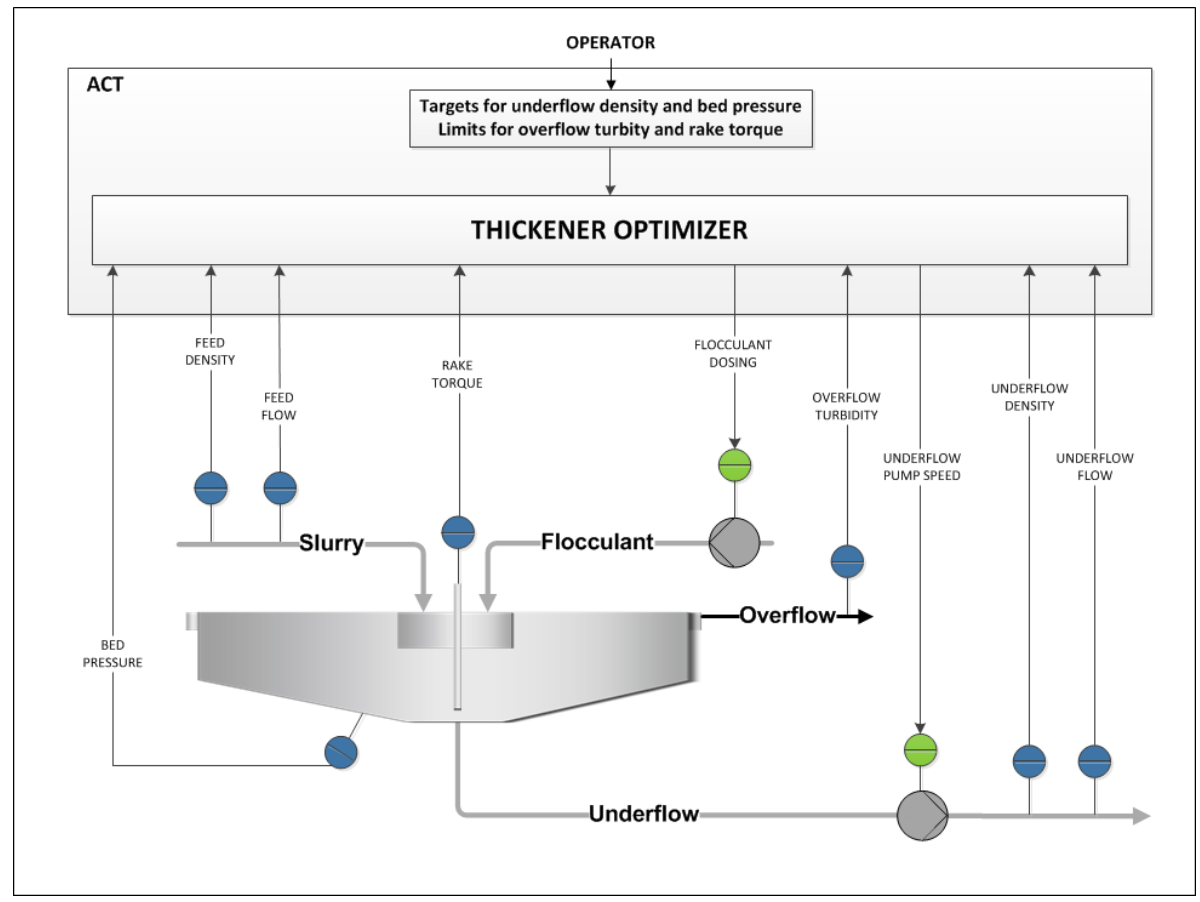

Figure 1 Optimizing control for thickeners - system structure

\subsection{Results from a nickel concentrate thickener}

To verify the potential of the optimizing control solution, it was implemented on a production scale nickel concentrate thickener. This concentrator plant has significant variation in the feed ore grade and ore hardness, which results in a varying tonnage of nickel concentrate produced. This results in varying feeds to the nickel concentrate, making the thickener difficult to run.

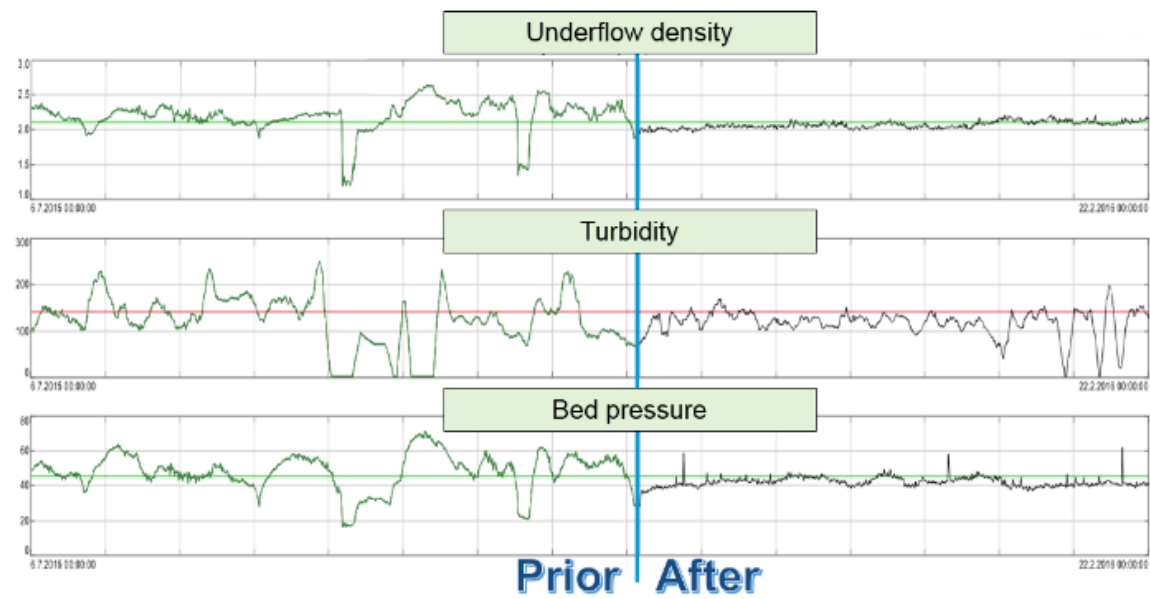

Figure 2 Thickening performance - Manual control versus optimizing control

Before the advanced control implementation, the thickener had traditional PI control loops for bed pressure and underflow density. However, these controllers were very rarely used due to difficult controller tuning. According to site personnel, it was very challenging to tune the PI loops to achieve reactivity to production changes and long-term stability. The outcome was that most of the operators were running the thickener on manual, meaning that a lot of operator attention was needed when production conditions changed.

As a result, the thickener was not able to maintain the required bed pressure and underflow density. Often, the thickener tended to run too empty, with the consequence that underflow density was lost and recirculation had to be turned on to increase the inventory level. Varying underflow density had a direct 
influence on concentrate filtration, because the decreased feed density decreased the filtration efficiency.

Another challenge caused by poor process controls emerged when more poorly settling material came into the thickener. If the operator was unable to immediately react to the situation with a polymer dosage, the material did not settle properly, resulting in a burst of solids to the overflow. Because the overflow was re-circulated backwards in the process as flotation spray water, the flotation performance was also disrupted by the solids covered with polymers.
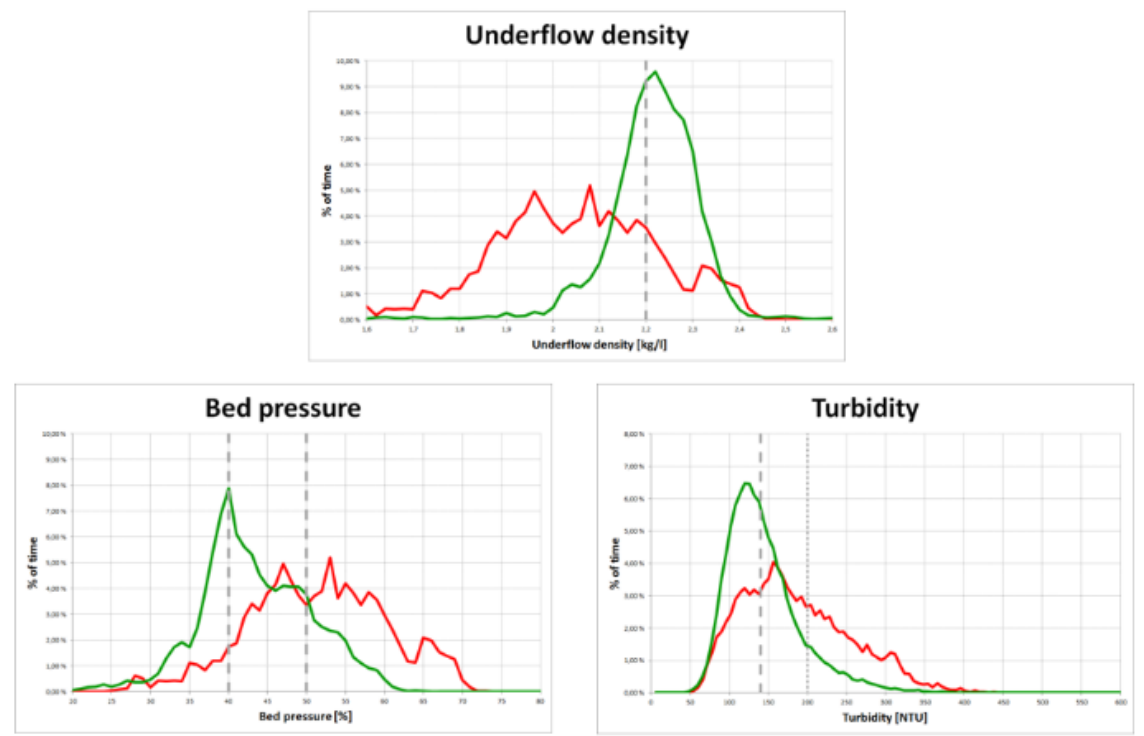

Figure 3 Relative distribution of underflow density, bed pressure and overflow turbidity - Manual control (red curve) versus optimizing control (green curve)

After commissioning, the performance of the optimizing control was compared to the situation with old controls (see Figures 2 and 3). When similar operating points were compared, it was evident that a remarkable improvement in process performance had been achieved (Figure 3). All in all, the optimizing solution was able to reduce the underflow density variation by $24 \%$, allowing the system to run with $6 \%$ higher average density. Overflow turbidity was controlled so that no major bursts of solids were caused to the circulating waters. Additionally, when the amount of solids in the overflow was below the set threshold, the controller decreased the polymer dosage, resulting in a decrease of approximately $10 \%$ in the average polymer consumption.

\section{$4 \quad$ Optimizing the performance of paste thickening}

In this chapter, the suitability of the optimizing control algorithm to control paste thickener is tested in a simulated process environment. In the beginning, a simple, heuristic model describing the main characteristic of paste thickening process is introduced. After this, results from test runs comparing the performance of optimizing control and traditional PI-controller setup are presented, and the suitability of the optimizing control for the paste thickening process was discussed.

\subsection{Simulation model for paste thickener}

In general, thickener can be considered as an integrator process, where the bed mass is dependent on the difference between input $Q_{\text {in }}$ and output $Q_{\text {out }}$ dry mass flows:

$$
\Delta Q=Q_{\text {in }}-Q_{\text {out }}=Q_{\text {in }}-Q_{\text {out }}^{U F}-Q_{\text {out }}^{\text {OF }} \approx Q_{\text {in }}-Q_{\text {out }}^{U F}
$$


Where the outgoing dry mass flow can be divided into the flow to the underflow $Q_{\text {out }}^{U F}$ and flow to the overflow $Q_{\text {out }}^{O F}$. In practice, $Q_{\text {out }}^{O F}$ is negligible when compared to the other mass flow rates, so that it can be ignored in the modeling.

Bed mass measurement is used to represent the inventory level of the thickener. In practice, it is a pressure measurement located in thickener underflow cone, which is calibrated to express the filling degree of the thickener. The response from the mass flow difference $\Delta Q$ to the change in bed mass $m$ can be represented with a simple integrator model

$$
\mathrm{G}_{m}(\mathrm{~s})=\frac{\mathrm{K}_{m}}{\mathrm{~s}} \mathrm{e}^{-\mathrm{d}_{m} \mathrm{~s}}
$$

Where $\mathrm{K}_{m}$ is process gain and $\mathrm{d}_{m}$ is process delay.

Phenomena influencing the formation of underflow density are very complicated. Obtained underflow density is dependent on the height of the solids bed, aggregate size and settling velocity of the solids material. These are instead dependent on the fluid shear rate, flocculant dosage, particle size distribution, and solids volumetric fraction (Remes and Vesa, 2014; Remes et al., 2009). For practical reasons, a straightforward approach was selected for our simulator, and it was assumed that the main influencing factors affecting the underflow density $\rho_{\mathrm{UF}}$ are the bed mass $m$ and the flocculant dosage $f_{\text {flocc }}$.

$$
\mathrm{G}_{\rho}(\mathrm{s})=\mathrm{G}_{\rho}^{\mathrm{m}}(\mathrm{s})+G_{\rho}^{\mathrm{flocc}}(\mathrm{s})
$$

The dynamic relationship between the bed mass and underflow density was expressed with a simple first order dynamic model.

$$
\mathrm{G}_{\rho}^{\mathrm{m}}(\mathrm{s})=\frac{\mathrm{K}_{\rho}^{\mathrm{m}}}{\tau_{\rho}^{\mathrm{m}} \mathrm{s}+1} \mathrm{e}^{-\mathrm{d}_{\rho}^{\mathrm{m}} \mathrm{s}}
$$

Where time constant $\tau_{\rho}^{\mathrm{m}}$ and delay $\mathrm{d}_{\rho}^{\mathrm{m}}$ are constants and the process gain $\mathrm{K}_{\rho}^{\mathrm{m}}$ has a logarithmic relationship to the bed mass $m$.

$$
\mathrm{K}_{\rho}^{\mathrm{m}}=\alpha \cdot \ln (\mathrm{m})+\beta
$$

Where $\alpha$ and $\beta$ are constants.

The influence of the flocculant dosage on underflow density was taken into account with a similar first order model.

$$
G_{\rho}^{\text {flocc }}(s)=\frac{\mathrm{K}_{\rho}^{\text {flocc }}}{\tau_{\rho}^{\text {flocc }} \mathrm{s}+1} \mathrm{e}^{-\mathrm{d}_{\rho}^{\text {flocc }} \mathrm{s}}
$$

Where all model parameters are constants. Correspondingly, similar linear first-order response models were also used to describe the dynamics responses from flocculant dosage and incoming dry mass flow to turbidity. Influence from underflow mass flow to overflow turbidity and from flocculant dosage to bed mass were assumed to be negligible.

Table 1 Key parameters of the simulated process

\begin{tabular}{cc}
\hline Variable & Value \\
\hline Thickener feed flow & $500 \sim 600 \mathrm{ton} / \mathrm{h}$ \\
Feed density & $45 \%$ \\
Thickener diameter & $30 \mathrm{~m}$ \\
Underflow density & $65 \sim 70 \%$ \\
Overflow turbidity & $130 \sim 150 \mathrm{NTU}$ \\
Time constant values & $2000 \sim 5000 \mathrm{~s}$ \\
Delay values & $300 \sim 4500 \mathrm{~s}$ \\
\hline
\end{tabular}


The model above was parameterized to match the presumed dynamics of the tailings paste thickener. Long delay and time constant values were used to emphasize the fact that paste thickeners are run with high inventory levels and high retention times, resulting in a sluggish and slowly turning process. The key running parameters of the simulator are presented in Table 1.

\subsection{Test bench setup}

Figure 4 shows the principle test system layout, which consisted of an industrial automation platform and a separate process simulator mimicking the real process. Both systems were implemented by using the Outotec Advanced Control Tools (ACT) environment. The control system and simulator were run as totally separate entities, and a similar interface was built between them like as is normally done between site DCS and ACT in real life installations. In the simulator, the heuristic model for paste thickener from previous chapter was implemented and a moderate amplitude white noise was added to "process measurements" to imitate the noise in real production processes.

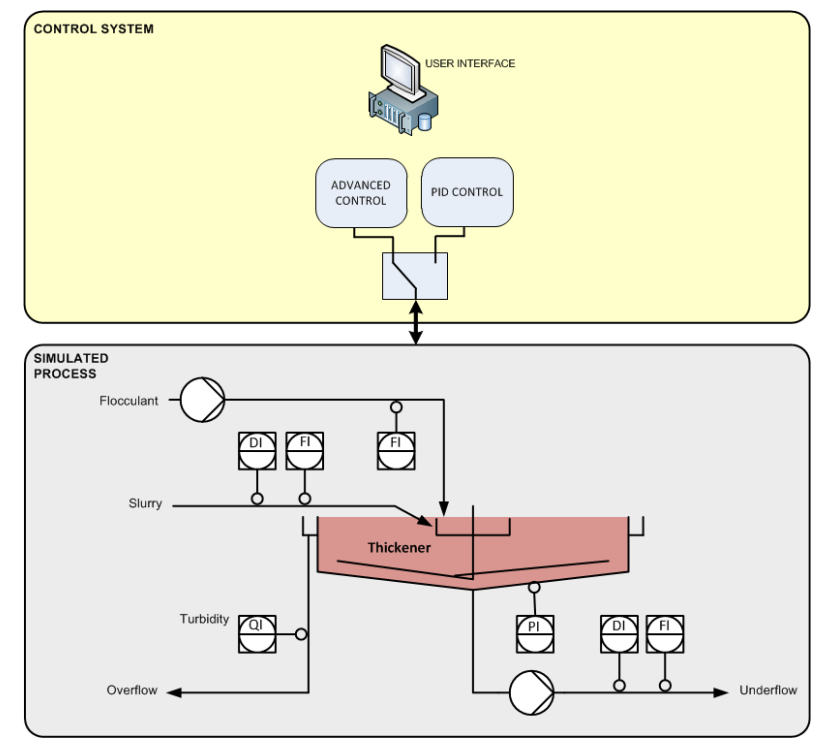

Figure 4 Test bench setup used for simulation runs

Two different control setups were tested with the simulator. The first one was the multivariable MPC concept presented in section 3 . The second was a traditional controller setup, where bed pressure was controlled by a PI-controller and the flocculant was dosed in proportion to thickener dry mass feed flow (see Figure 5). Both control system setups were taken into use in a similar way normally done in real life installations. Process step response tests were performed to model the process behavior, and the models for MPC were built with the modeling tools integrated in the ACT environment. Finally, both controllers were tuned so that the fastest possible closed loop responses were obtained with a modest amount of overshooting.

\subsection{Comparison of traditional and optimizing control strategies}

In the first trial, the objective was to monitor the closed loop response of both systems by doing a stepwise $+1 \%$ change in underflow density. Because the optimizing control has the density as one controlled variable, a direct change to the density target value was possible. In addition, the other target values given to the controller were the preferred bed pressure operating zone and maximum acceptable turbidity. 

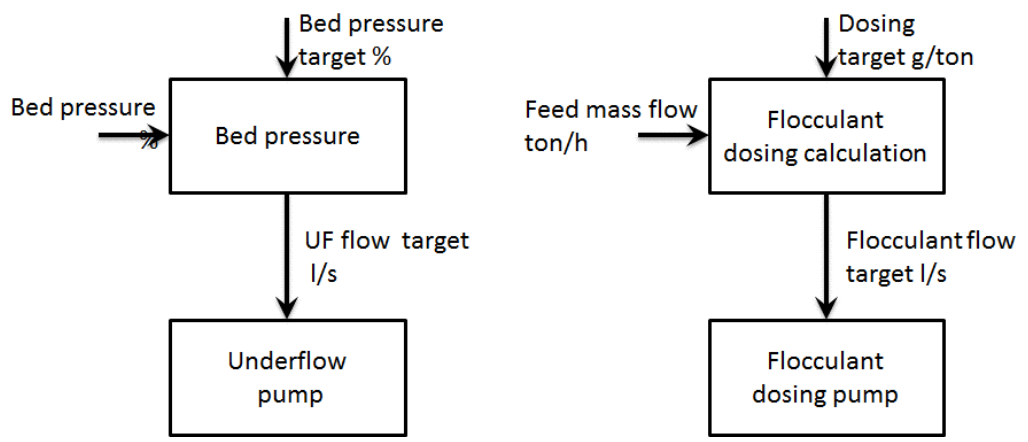

Figure 5 Traditional thickener controller setup

With traditional controller, only the bed pressure has a target value. Therefore, an equivalent change to $1 \%$ density change has to be made to the bed pressure target value. Because the bed pressure density relationship is not linear and is also dependent on the process operating point, running a certain bed level target does not guarantee a certain density. Therefore, in this concept an error always exists between the targeted and actual underflow density.

Results from the first trial run are shown in Figures 6 and 7. Because both controllers were tuned to deliver a similar, smooth response, the initial response is similar with both controller setups. However, in the longer run the optimizing controller is clearly better in smoothing out the transient, while the PI controller continues oscillating near the target value. The reason for this is that systems having a long delay and a long time constant are difficult for traditional PI controllers, and therefore a tradeoff has to be made between system robustness and speed of controller response.

With the model-based controller, this sort of trade-off is not required, because the controller is inherently capable of handling the complicated process dynamics. In addition, the controller can simultaneously control both underflow density and inventory levels, so that highly accurate density control is obtained.

The target for the second test was to examine how well the systems are able to reject process disturbances. This was done by making a sudden, stepwise $+10 \%$ ( +60 ton/h) change to the thickener feed flow. No changes to controller target values were made. Results from the second trial are shown in Figures 8 and 9.

This sort of sudden substantial feed change is very difficult for the controls when totally unexpected. Because the simulated paste thickener is a very slow process, it takes a long time before the change in feed is noted in the bed pressure or density measurement. If the controller has information from the feed line, more timely corrections can be made and smaller disturbance is caused to the thickening process. This can be clearly seen from responses below. Because the optimizing control is using the flow and density information from the feed line, more timely and precise control actions are implemented. When we combine this with the clearly better overall dynamic behavior of the optimizing controller, a superior disturbance rejection is obtained in comparison to the traditional controller setup. 

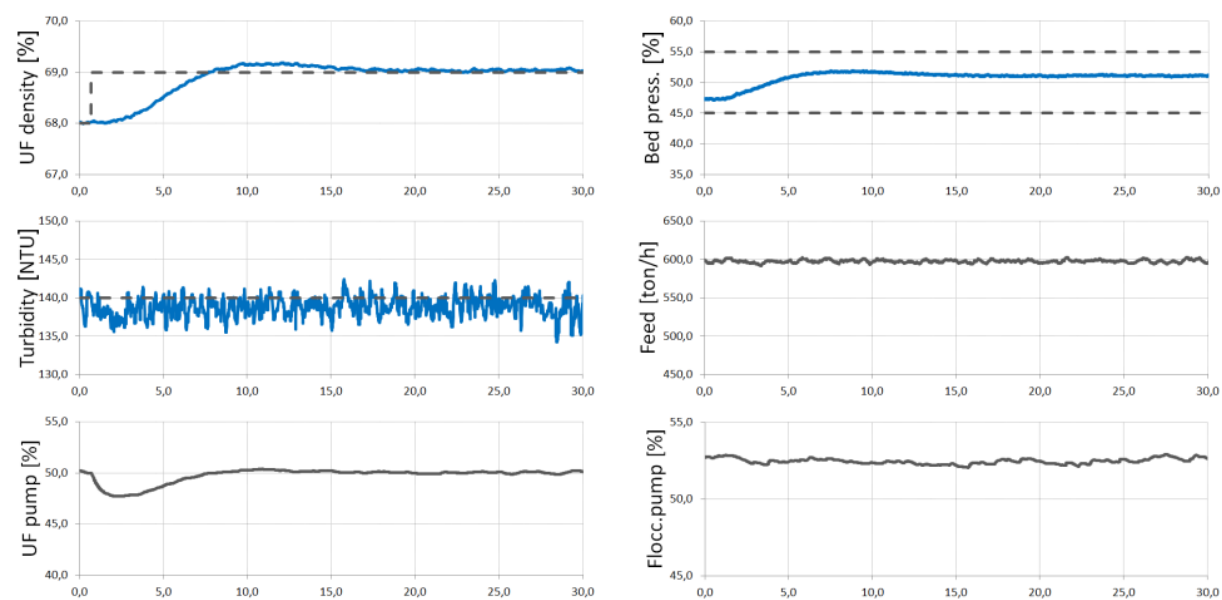

Figure 6 Underflow density change with optimizing controller (solid line $=$ measured, dashed line $=$ target value)
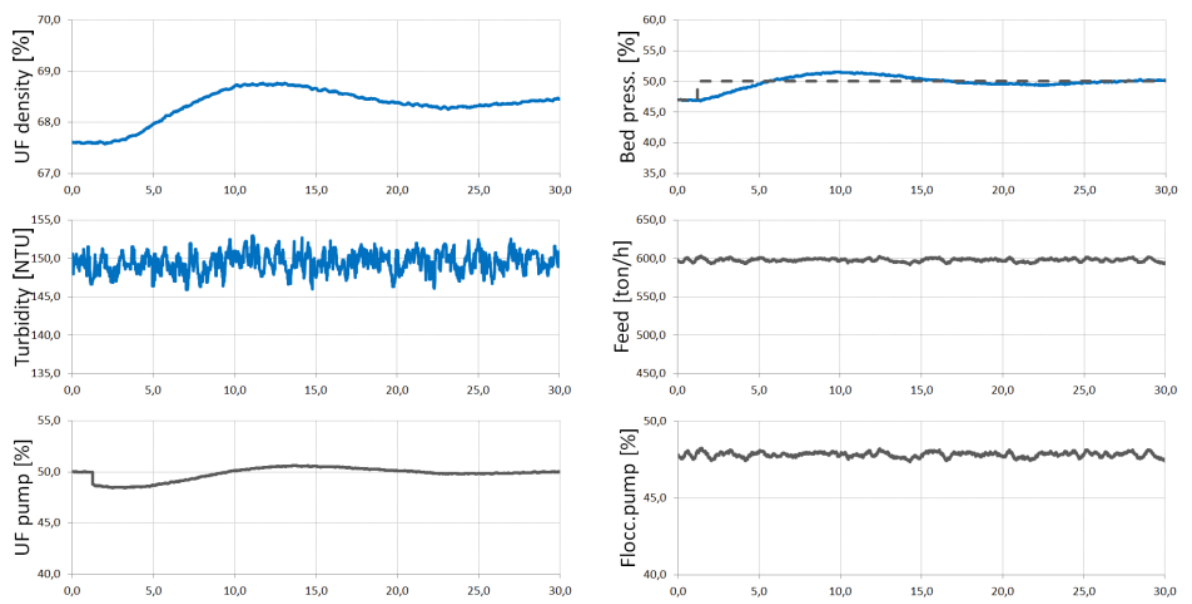

Figure 7 Underflow density change with traditional controller (solid line $=$ measured, dashed line $=$ target value) In traditional control, the flocculant is dosed in relation to the incoming mass flow: when the feed flow is changed, an instantaneous change in flocculant dosage is obtained accordingly. If there is excessive amount of fine solids, they will escape to the overflow, in the manner shown in Figure 9. The optimizing control controls the overflow turbidity instead, so that it is always ensured that the required overflow quality is achieved. On the other hand, if the material settles easily and turbidity is well below the limit value, flocculant dosage can be reduced to gain chemical savings.
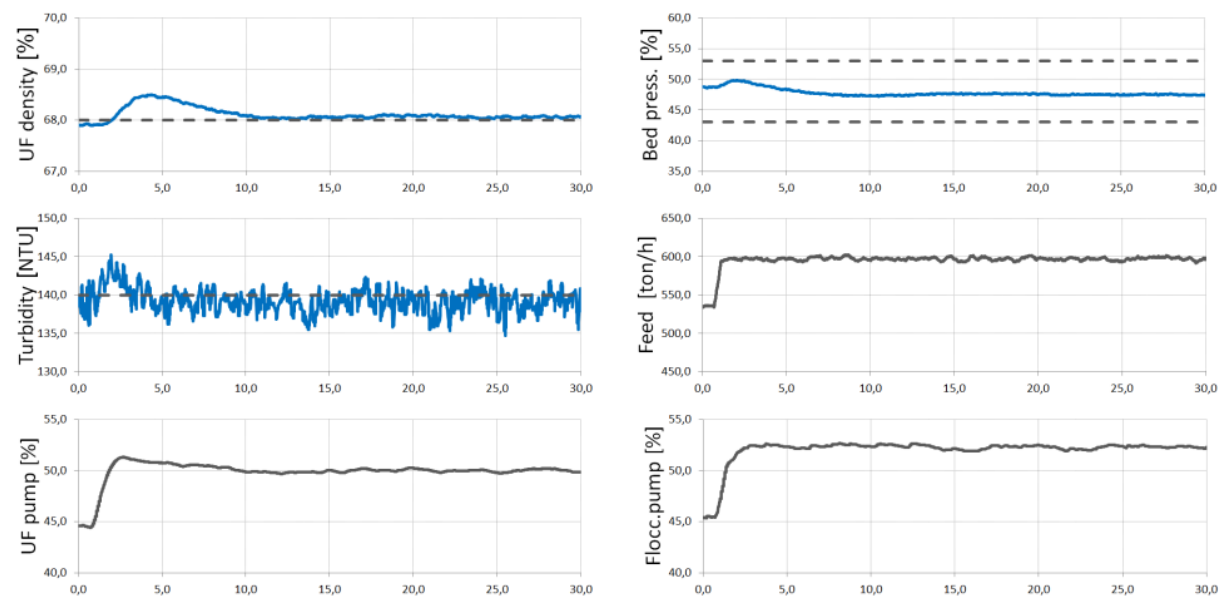

Figure 8 Feed change behavior with the optimizing controller (solid line = measured, dashed line = target value) 

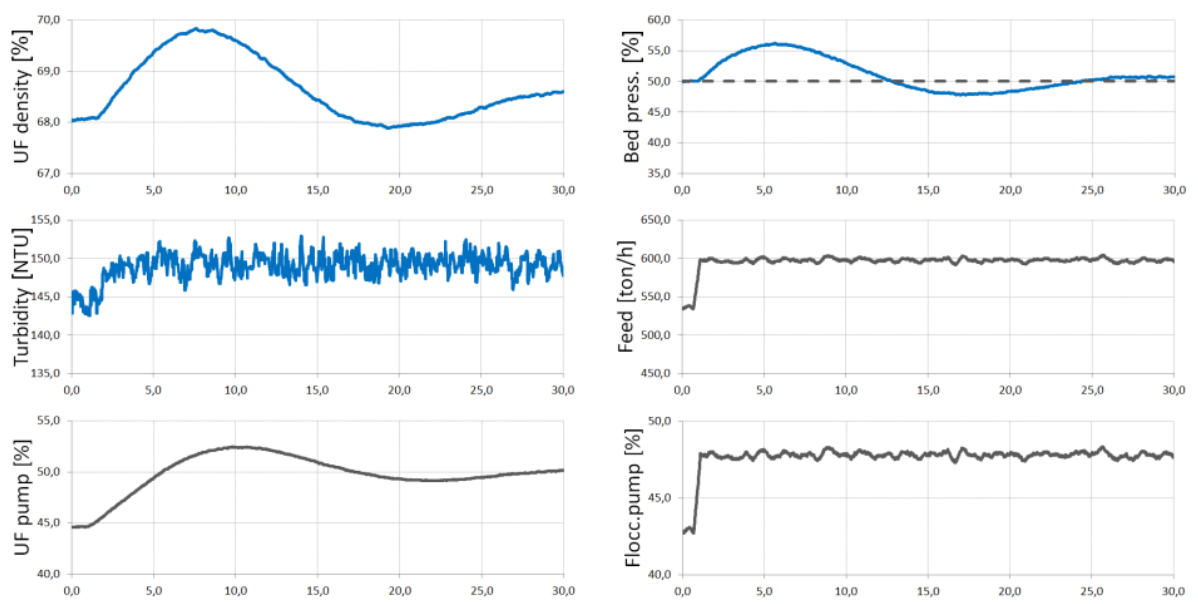

Figure 9 Feed change behavior with the traditional controller (solid line $=$ measured, dashed line $=$ target value)

\section{$5 \quad$ Conclusions}

Traditionally, thickener controls have been built by using single loop Proportional Integral (PI) controllers in the plant's DCS/PLC systems. Based on our experiences from numerous thickener installations, many of these implementations suffer from poor process control results. One of the main reasons for this is that traditional single loop controllers are not optimal to handle the slow and complicated process dynamics.

To overcome these limitations, a novel approach to utilize multivariable model predictive control (MPC) to control thickeners was presented in this paper. This application integrates highly interactive controls of thickener inventory level, underflow density and overflow clarity into a single multivariable control system. The MPC approach has the inbuilt ability to take changes in the incoming flow into account, deal with process constraints, and support prioritization between the controlled variables.

Based on the results from the production scale concentrate thickener and the simulation results presented in this paper, this system provides an efficient tool for process optimization. The thickener can consistently run with higher and more stable underflow density, so that required level of overflow clarity is simultaneously obtained. For paste thickeners, this provides an efficient tool to ensure the operation in the desired operation window and better tolerance with regard to feed disturbances.

\section{References}

Kosonen, M. 2004, 'Modeling and online control of paper machine wire retention', Dissertation, Tampere University of Technology, Tampere, Finland.

Maciejowski, J.M. 2002, 'Predictive control with constraints', Prentice Hall, London.

Parker, B., Rivett, T., Backeberg, T., McIntosh, A., El-Masry, S. and Heath, A. 2016, 'Debottlenecking of thickeners in a changing environment', Outotec white paper.

Remes, A., Aaltonen, J., Koivo, H. 2009, 'Soft-sensor estimation of an apatite thickener operation at the Siilinjärvi concentrator', Preprints of IFAC Workshop on Automation in Mining, Minerals and Metal Industry, Nina del Mar, Chile

Remes, A., Vesa, L. 2014, 'Thickener modeling - from laboratory test work to plant scale dynamics simulation', IMPC 2014, Santiago, Chile 